\title{
Alleviative effect of grape seed proanthocyanidin extract on small artery vascular remodeling in spontaneous hypertensive rats via inhibition of collagen hyperplasia
}

\author{
YING LIANG $^{1,2}$, HAIQING GAO ${ }^{1}$, JIAN WANG ${ }^{1}$, \\ QUANZHEN WANG $^{1}$, SHAOHUA ZHAO ${ }^{1}$, JUN ZHANG ${ }^{1}$ and JIE QIU ${ }^{1}$ \\ ${ }^{1}$ Department of Geriatric Cardiology, Qilu Hospital of Shandong University, Jinan, Shandong 250012; \\ ${ }^{2}$ Department of Geriatric Cardiology, Qianfoshan Hospital of Shandong University, Jinan, Shandong 250013, P.R. China
}

Received October 18, 2015; Accepted October 6, 2016

DOI: $10.3892 / \mathrm{mmr} .2017 .6292$

\begin{abstract}
Vascular remodeling is a primary contributor to the initiation and development of hypertension, which has a pathological association with subsequent multi-organ damage. Grape seed proanthocyanidin extracts (GSPE) exhibit protective cardiovascular effects, resulting from their anti-oxidant and anti-inflammatory properties. However, the function and mechanism underlying the effect of GSPE on small artery remodeling remain to be elucidated. The present study investigated the effect of GSPE on vascular remodeling in the mesenteric small arteries of spontaneous hypertensive rats (SHR). Parameters associated with hypertension, including systolic blood pressure, oxidative stress, morphological and ultrastructural alteration of vessels, deposition of collagen and transforming growth factor (TGF)- $\beta 1$, were analyzed. The results revealed that GSPE alleviated hypertension-induced hypertrophic vascular remodeling in the small arteries of SHR, which was independent of blood pressure. GSPE decreased oxidative stress associated with hypertension in SHR and suppressed the increased expression of TGF- $\beta 1$,
\end{abstract}

Correspondence to: Dr Haiqing Gao or Dr Jie Qiu, Department of Geriatric Cardiology, Qilu Hospital of Shandong University, 107 Wenhua Xi Road, Jinan, Shandong 250012, P.R. China

E-mail: gaohaiqing52@hotmail.com

E-mail: qiujie0408@yahoo.com.cn

Abbreviations: GSPE, grape seed proanthocyanidin extract; TGF, transforming growth factor; SHR, spontaneous hypertensive rat; AF, adventitia fibroblasts; VSMC, vascular smooth muscle cell; ECM, extracellular matrix; WKY, Wistar-Kyoto rats; SBP, systolic blood pressure; MDA, malondialdehyde; ROS, reactive oxygen species; SOD, superoxide dismutase; CAT, catalase; ID, inner diameter; VCSA, vascular cross-sectional area; WCSA, wall cross-sectional area; LCSA, luminal cross-sectional area; WT, wall thickness; CSA, cross-sectional area

Key words: grape seed proanthocyanidin extracts, hypertension, small artery remodeling, collagen, adventitia fibroblasts which blocked the translocation and differentiation of adventitia fibroblasts and eventually inhibited collagen hyperplasia in the blood vessel. The inhibitory effect of GSPE on small artery remodeling was achieved via its suppressive effect on oxidant production and the subsequent intercellular and intracellular cascades. The findings of the present study supported the potential therapeutic value of GSPE for the treatment of hypertension.

\section{Introduction}

Vascular remodeling is recognized as a primary contributor to the damage of target organs in the development of hypertension (1). Artery walls that have undergone hypertension are identified by the increased size of the vascular wall, particularly the tunica media, which is accompanied by a narrowed lumen. Proliferation of vascular smooth muscle cells (VSMC) occurs, along with alterations to the elastic properties of the arterial wall (2). In the vascular wall extracellular matrix (ECM), the abnormal deposition of collagen contributes to vascular remodeling (3). Reactive oxygen species (ROS) are believed to be involved in the development of hypertension via the promotion of vascular remodeling (4), which induces collagen hyperplasia in the vascular wall (5). GSPE is an anti-free radical reagent (6), which may be beneficial for the treatment of cardiovascular diseases, including arteriosclerosis, hypertension and subsequent organ damage (7). ROS are second messengers that regulate cellular function via reversibly oxidizing specific amino acid residues of key target proteins (8), adversely affecting various important cell signaling pathways, including the transforming growth factor (TGF)- $\beta 1$ signaling cascade and the expression of ECM (9). The present study hypothesized that GSPE may have a therapeutic effect on vascular remodeling associated with collagen accumulation in the mesenteric small arteries of spontaneous hypertensive rats (SHR). The understanding of the association between the anti-oxidative effect of GSPE and the underlying mechanisms involved in vascular remodeling may support its use as a potential therapeutic agent for the treatment of small vascular remodeling in cardiovascular disease. 


\section{Materials and methods}

Animal model. All experimental procedures were approved by the Animal Experiment Ethics Review Committee of Shandong University, (Jinan, China). At total of 20 SHR and 10 Wistar-Kyoto (WKY) rats (age, 20 weeks; weight, $369.25 \pm 12.11 \mathrm{~g}$ ) were obtained from Vital River Laboratory Animal Technology Co., Ltd. (Beijing, China). Rats were maintained in individual cages at $22 \pm 2^{\circ} \mathrm{C}, 50-55 \%$ humidity, with $12 \mathrm{~h} \mathrm{light/dark} \mathrm{cycles.} \mathrm{They} \mathrm{were} \mathrm{fed} \mathrm{with} \mathrm{standard}$ rat chow and had free access to tap water for 1 week. Rats were subsequently randomly assigned to three groups $(n=10)$ : i) WKY control (WKY), ii) spontaneous hypertension control (SHR-C), and iii) spontaneous hypertension with GSPE treatment (SHR-T). Rats in the WKY and SHR-C groups were orally administered $1.0 \mathrm{ml}$ saline per day, where as those in the SHR-T group were treated with $250 \mathrm{mg} / \mathrm{kg} /$ day GSPE (lot no. 002-1312065-13; Natural Product R\&D Co., Ltd., Tianjin, China) dissolved in $1.0 \mathrm{ml}$ saline. At the end of week 22, rats were sacrificed following anesthesia with $10 \%$ chloral hydrate $(300 \mathrm{mg} / \mathrm{kg}$ ) by intraperitoneal injection provided by Qilu Hospital of Shandong University (Jinan, China). Serum from peripheral blood obtained from the abdominal aorta and the harvested mesenteric small arteries were stored at $-80^{\circ} \mathrm{C}$.

Systolic blood pressure (SBP). Tail SBPs were measured prior to experimentation, and subsequently once a week until the end of the experiment using a non-invasive sphygmomanometer (Softron tail-cuff; Softron Beijing Incorporated, Beijing, China). All rats were conscious and free when measurements were obtained.

ELISA. Malondialdehyde (MDA), $\mathrm{H}_{2} \mathrm{O}_{2}$, ROS, superoxide dismutase (SOD), and catalase (CAT) levels in mesenteric artery tissues were measured using the following oxidative stress ELISA kits: $\mathrm{H}_{2} \mathrm{O}_{2}$ assay kit (cat. no. 20131220; absorbance reading at $405 \mathrm{~nm}$ ), ROS assay kit (cat.no. 20131126; absorbance reading at $525 \mathrm{~nm}$ ), SOD assay kit (cat. no. 20131120; absorbance reading at $450 \mathrm{~nm}$ ), CAT assay kit (cat. no. 20131218; absorbance reading at $405 \mathrm{~nm}$ ) and Microscale MDA assay kit (cat. no. 20131112; absorbance reading at $523 \mathrm{~nm}$ ), which were purchased from Nanjing Jiancheng Bioengineering Institute (Nanjing, China) according to the manufacturer's protocol.

Histopathology. Mesenteric small artery samples for protein and RNA detection were washed in $4^{\circ} \mathrm{C}$ saline and frozen in liquid nitrogen followed by storage at $-80^{\circ} \mathrm{C}$. Segments for electron microscopy were immediately cut into $1 \times 1 \times 1 \mathrm{~mm}$ sections and fixed in $2.5 \%$ glutaraldehyde solution. Fixed sections were processed with $10 \%$ formalin and embedded in paraffin. Vascular tissue slices were stained with Sirius red. Pathological alterations were observed and analyzed in 5 randomly selected images per section using the Olympus DP71 imaging system (Olympus Corporation, Tokyo, Japan) and Image-Pro Plus software 5.0 (Media Cybernetics, Inc., Rockville, MD, USA). The inner diameter (ID), vascular cross-sectional area (VCSA), wall cross-sectional area (WCSA), luminal cross-sectional area (LCSA) and wall thickness (WT) of the vascular tissue were measured. The ratios of WT to ID (WT/ID) and WCSA to LCSA (WCSA/LCSA) were subsequently calculated.
Type I and III collagen and elastin were stained with Sirius red-victoria blue (10) and were observed using polarization microscopy. The area and ratio of elastin and collagen were calculated using Image Pro-Plus software in 5 different fields.

Western blotting. Total protein was extracted from the small mesenteric arterial tissue. Tissues were washed twice with cold phosphate buffered saline and were homogenized with ice-cold cell radio immunoprecipitation assay lysis buffer (cat. no. P0013B; Beyotime Institute of Biotechnology, Haimen, China) for $30 \mathrm{~min}$ on ice and the supernatant was collected following centrifugation $(14,000 \times g$ for $15 \mathrm{~min})$ at $4^{\circ} \mathrm{C}$. The concentration of total protein was measured using a bicinchoninic acid protein assay kit (cat. no. P0006; Beyotime Institute of Biotechnology). An equal quantity of protein $(60 \mu \mathrm{g})$ was loaded onto SDS-PAGE gels (12\% Mini-PROTEAN ${ }^{\circledR}$ Gel; cat. no. 456-1094; Bio-Rad Laboratories, Inc., Hercules, CA, USA). The proteins were transferred to polyvinylidene difluoride membranes which was monitored by Ponceau $\mathrm{S}$ staining. Membranes were blocked with blocking buffer [5\% fat free milk in TBS plus $0.1 \%$ Tween-20 (TBST), $\mathrm{pH}$ 7.5] at room temperature for $1 \mathrm{~h}$ and then incubated for $2 \mathrm{~h}$ with the following primary antibodies: Mouse-anti-TGF- $\beta 1$ (cat. no. ab64715; dilution, 1:500; Abcam, Cambridge, MA, USA), rabbit-anti-collagen I (cat. no. ab96723; dilution, 1:500; Abcam), rabbit-anti-collagen III (cat. no. 13548-1; dilution, 1:400; Protein Tech Group, Inc., Chicago, IL, USA) and mouse-anti-actin (cat. no. ab3280; dilution, 1:2,000; Abcam) at $4^{\circ} \mathrm{C}$ overnight. Following 3 washes with TBST (10 min each), membranes were incubated with a species-specific horseradish peroxidase-conjugated secondary antibodies: Goat anti-rabbit (cat. no. ZB-2301; dilution, 1:5,000; ZSGB-BIO, Beijing, China) and goat anti-mouse (cat. no. Zf-0312; dilution, 1:5,000; ZSGB-BIO) at room temperature for $2 \mathrm{~h}$. Following 3 washes, membranes were soaked in Chemiluminescence Detection reagent (EMD Millipore, Billerica, MA, USA) and protein bands were visualized using an ImageQuant LAS-4000 imaging system (GE Healthcare Life Sciences, Chalfont, UK). Quantitative analysis was performed using AlphaEaseFC analysis software 4.1.0 (Alpha Innotech, San Leandro, CA, USA) and normalized to densitometric values of $\beta$-actin.

Reverse transcription-quantitative polymerase chain reaction $(R T-q P C R)$. Total RNA was extracted from small mesenteric arterial tissue using TRIzol ${ }^{\circledast}$ reagent (Invitrogen; Thermo Fisher Scientific, Inc., Waltham, MA, USA) according to the manufacturer's protocol. First-strand cDNA was obtained using the RevertAid First Strand cDNA Synthesis kit (Thermo Fisher Scientific, Inc.) with oligo-d(T) as an initial primer. qPCR was performed using a standard TaqMan ${ }^{\circledR}$ PCR kit (Applied Biosystems; Thermo Fisher Scientific, Inc.) with the obtained cDNA on an ABI PRISM ${ }^{\circledR} 7300$ PCR instrument (Applied Biosystems; Thermo Fisher Scientific, Inc.) in a $25 \mu 1$ reaction volume containing Maxima SYBR-Green qPCR Master Mix (2X) (Applied Biosystems; Thermo Fisher Scientific, Inc.), cDNA and primers. Primer sequences were as follows: Forward, 5'-CTGCTGACCCCCACTGATAC-3' and reverse, 5'-GTGAGCACTGAAGCGAAAGC-3' for TGF- $\beta 1$; forward, 5'-GGCATAAAGGGTCATCGTG-3' and reverse, 5'-GAACCTTCGCTTCCATACTC-3' for collagen I; forward, 
A

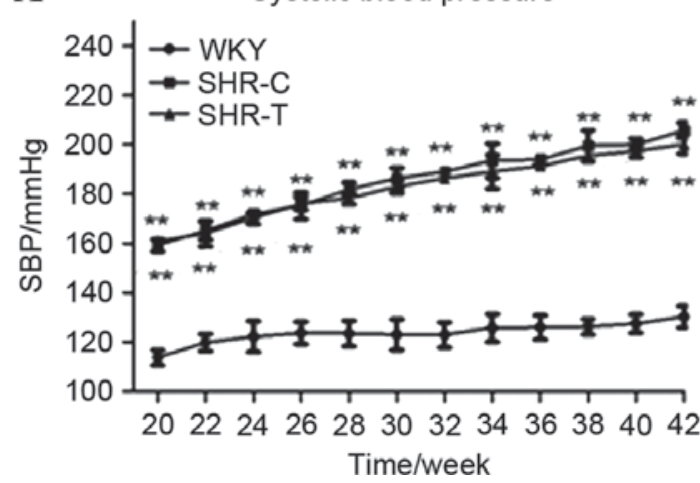

B

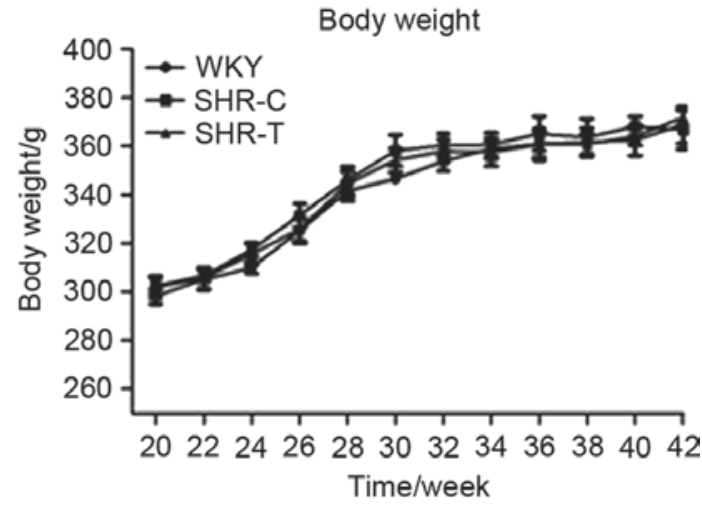

Figure 1. Effect of GSPE on SBP and body weight. (A) SBP was significantly increased in the SHR-C group compared with the WKY group. GSPE did not significantly alter SBP compared with SHR-C group. (B) No significant alterations in weight were observed during the experiment. Data are expressed as the mean \pm standard error $(n=10)$. ${ }^{* *} \mathrm{P}<0.01$ vs. WKY group. GSPE, grape seed proanthocyanidin extract; SBP, systolic blood pressure; SHR, spontaneous hypertension rat; T, treatment; C, control; WKY, Wistar-Kyoto.

5'-AGATCATGTCTTCACTCAAGTC-3' and reverse, 5'-TTT ACATTGCCATTGGCCTAG-3' for collagen III; forward, 5'-CGGGAAATCGTGCGTGAC-3' and reverse, 5'-TCGCTC CAACCGACTGCT-3' for $\beta$-actin. Thermocycling conditions were set at $95^{\circ} \mathrm{C}$ for $20 \mathrm{sec}$, followed by $60^{\circ} \mathrm{C}$ for $20 \mathrm{sec}$; 40 cycles were performed. The gene expression was relatively quantified (11) and normalized by the expression of $\beta$-actin which was performed according to the manufacturer's protocol and the article (12).

Ultrastructure. The mesenteric arterial tissue was fixed with $3 \%$ glutaraldehyde in cacodylate buffer at $4^{\circ} \mathrm{C}$ for $2 \mathrm{~h}$ and subsequently post fixed in a $1 \%$ osmium-tetroxide phosphate buffer solution for $1 \mathrm{~h}$. Dehydrated tissue sections were embedded in epoxy resin and were sliced into 75-nm semi-thin sections, which were stained with uranylacetate and lead citrate. Slides were observed under an H-800 transmission electron microscope (Hitachi, Ltd., Tokyo, Japan).

Statistical analysis. A one-way analysis of variance was performed using SPSS software 17.0 (SPSS, Inc., Chicago, IL, USA), followed by a Student-Newman-Keuls or least significant difference post-hoc test. Results are presented as the mean \pm standard deviation. $\mathrm{P}<0.05$ was considered to indicate a statistically significant difference and the null hypothesis was that there were no differences between the groups.

\section{Results}

Systolic blood pressure and body weight. SBP increased with age in all groups, and was significantly increased in the SHR-C compared with the WKY group $(\mathrm{P}<0.01$; Fig. 1A). Treatment with GSPE did not significantly alter SBP compared with the SHR-C group $(\mathrm{P}>0.5)$. No significant weight alterations were observed between the groups at any time ( $\mathrm{P}>0.5$; Fig. 1B).

Anti-oxidative stress effect of GSPE in spontaneous hypertension. Hypertensive rats exhibited significantly increased levels of ROS (P<0.01; Fig. 2A), $\mathrm{H}_{2} \mathrm{O}_{2}(\mathrm{P}<0.01$; Fig. 2B) and MDA $(\mathrm{P}<0.01$; Fig. $2 \mathrm{C})$, compared with the WKY group. However, this increase was partially reversed by treatment with GSPE $(\mathrm{P}<0.05)$. The levels of the oxidative enzymes, SOD $(\mathrm{P}<0.01$;
Fig. 2D) and CAT ( $\mathrm{P}<0.01$; Fig. 2E) were decreased in SHR-Compared with WKY controls; however, this decrease was partially reversed by GSPE $(\mathrm{P}<0.05)$.

Effect of GSPE on morphological alterations of small arteries. Vascular tissue slices were stained with Sirius red-victoria blue and pathological changes were observed using polarization microscopy primarily under a brightfield. In WKY, the typical three-layer structure was observed. The inner layer was thinner and the elastic lamina between the endangium and media was dyed green-blue. VSMCs were arranged as a circular multilayer in the media and collagens were observed as red, primarily resident in the adventitia (Fig. 3A). In SHR-C, broken endangium and collagen fibers in the lumen were presented in images captured under a brightfield, and the small mesenteric arteries had become narrowed and thickened. Disarranged collagen was observed in the media and adventitia (Fig. 3B). Following GSPE treatment, wall thickening and luminal stenosis of small mesenteric arteries were reduced. In addition, collagen levels were reduced, particularly in the media (Fig. 3C).

Effect of GSPE on collagen expression and distribution in small mesenteric arteries. Type I/III collagen and elastin were stained with Sirius red-victoria blue and were observed using polarization microscopy. Collagen was observed as red and elastin was observed as green-blue, under a brightfield. Under a darkfield, type I collagen appeared as red/orange fibers with strong double refraction, whereas type III collagen appeared as thin green fibers with weak birefringence. In WKY, type I collagen was primarily located in the media and type III collagen primarily appeared in the adventitia, with reduced levels present in the media (Fig. 3D). There were no collagen fibers present in the inner layer of the small artery. However, in the SHR-C small mesenteric artery, an overload of disarranged collagen was observed in the media and adventitia. In addition, collagen fibers were observed in the inner layer (Fig. 3E). Following, GSPE treatment, no collagen fibers were observed in the inner layer (Fig. 3F). Type I and III collagen were reduced in the media compared with the SHR-C group.

Quantification of pathological alterations. Image-ProPlus software was used to analyze microscope images and to 

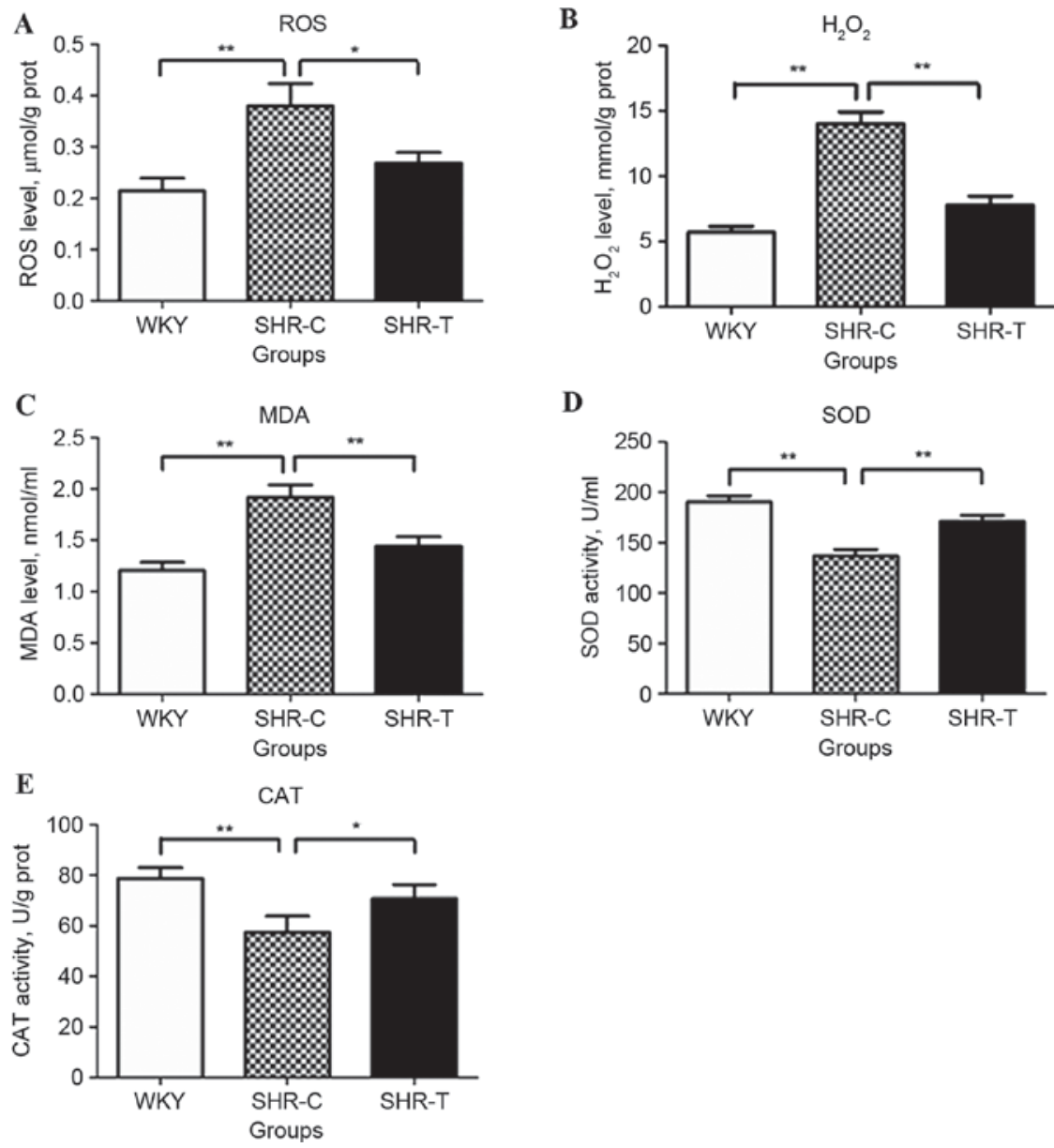

Figure 2. Effect of GSPE on ROS, $\mathrm{H}_{2} \mathrm{O}_{2}$, MDA, SOD, and CAT levels in mesenteric arterial tissues. Hypertensive rats exhibited a significantly increased level of (A) ROS, (B) $\mathrm{H}_{2} \mathrm{O}_{2}$ and (C) MDA compared with the WKY control group. This was partially reversed by treatment with GSPE. The levels of the oxidative enzymes (D) SOD and (E) CAT were decreased in hypertensive subjects; however, this was attenuated by administration of GSPE. "P $<0.05$ and ${ }^{* *} \mathrm{P}<0.01$. GSPE, grape seed proanthocyanidin extract; SHR, spontaneous hypertension rat; T, treatment; C, control; WKY, Wistar-Kyoto; ROS, reactive oxygen species, $\mathrm{H}_{2} \mathrm{O}_{2}$, hydrogen peroxide; MDA, malondialdehyde; SOD, superoxide dismutase; CAT, catalase.
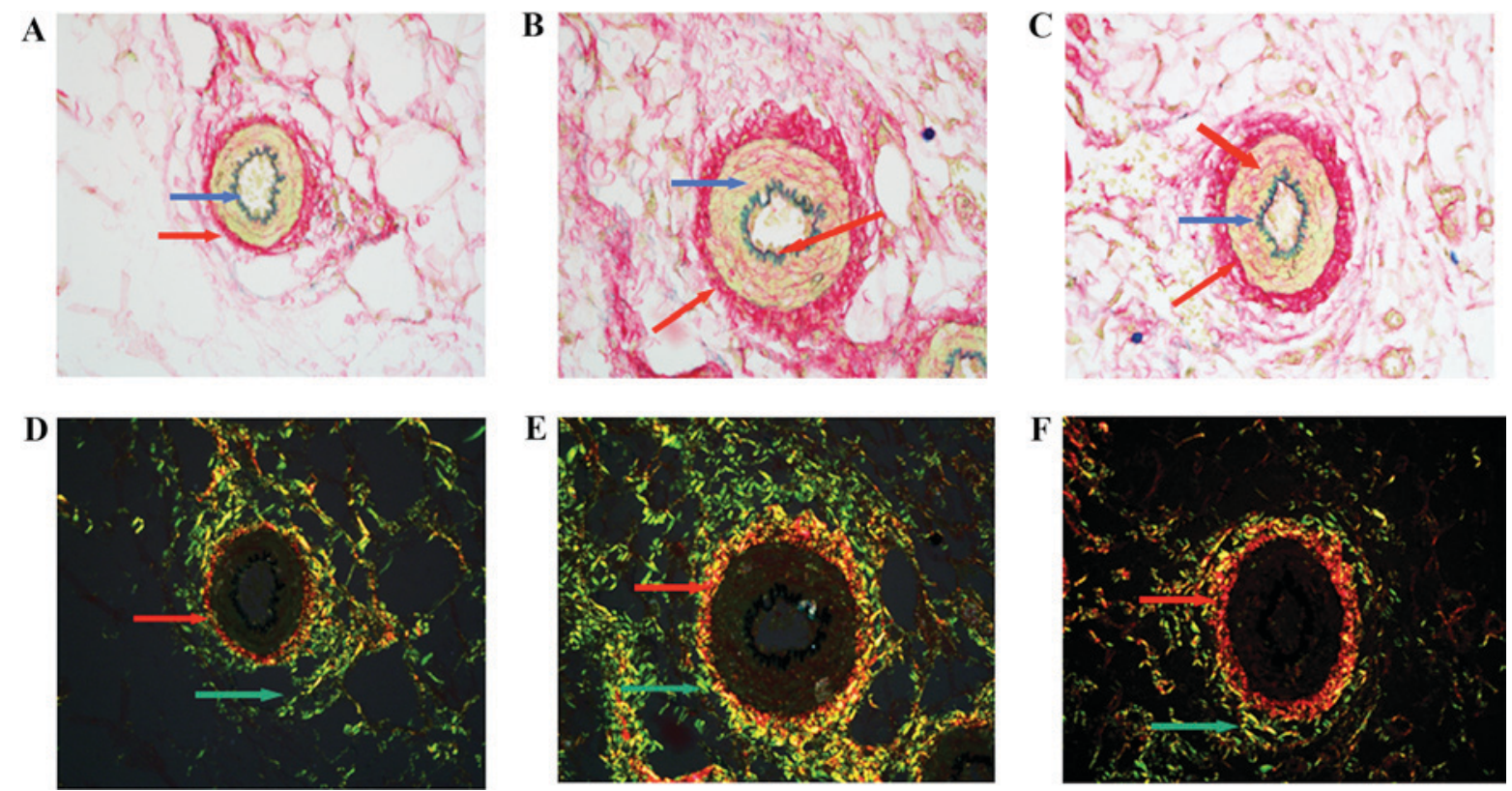

Figure 3. Effect of GSPE on the morphological alterations of the small mesenteric artery. Sirius red-victoria blue staining of small mesenteric arterial tissue from (A) WKY, (B) SHR-C and (C) SHR-T groups, observed under a brightfield. Collagen was stained red and is indicated by red arrows. Elastin was dyed green-blue and is indicated by blue arrows. Sirius red-victoria blue staining of small mesenteric arterial tissue from (D) WKY, (E) SHR-C and (F) SHR-T groups, observed under a darkfield. Collagen I was red/orange and is indicated by red arrows. Collagen III was green and is indicated by green arrows. GSPE, grape seed proanthocyanidin extract; SHR, spontaneous hypertension rat; T, treatment; C, control; WKY, Wistar-Kyoto. 
A

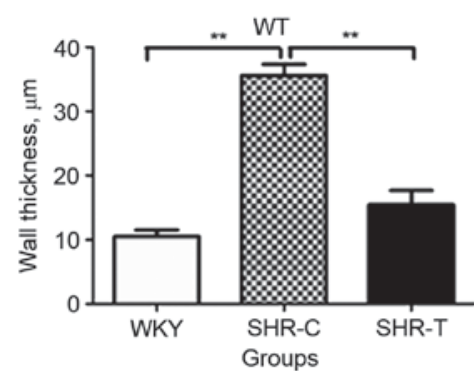

C

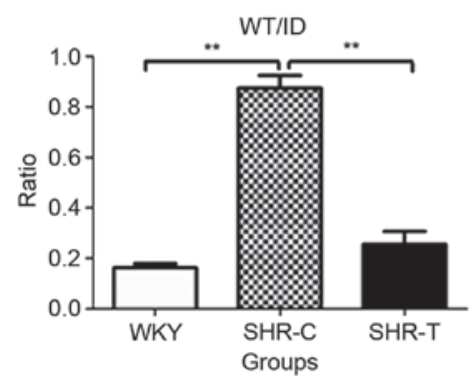

E

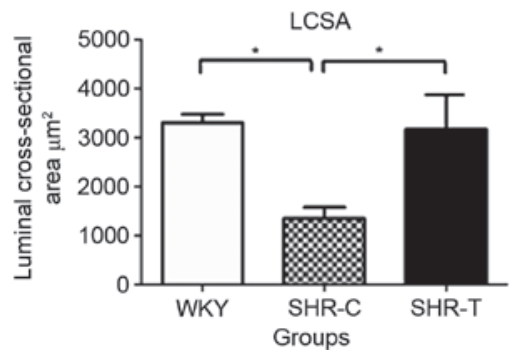

B

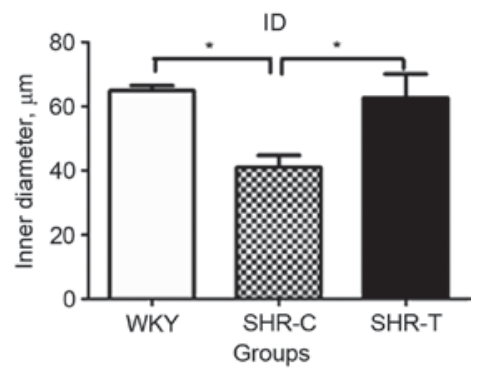

D
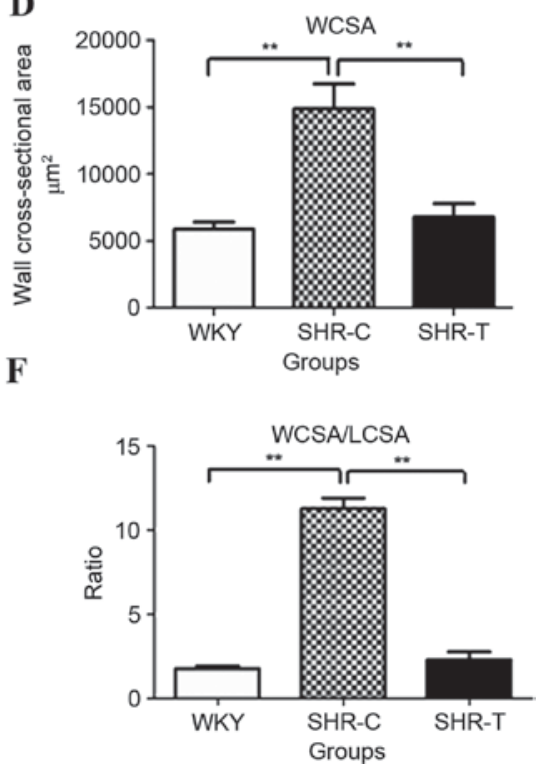

Figure 4. Effect of GSPE on vascular geometrical measurement of small mesenteric arteries. The diameter of vascular wall and lumen and the thickness of vascular wall was measured in Sirius red-victoria blue stained sections. (A) WT was increased in the SHR-C group compared with the WKY group, and this was significantly reversed by treatment with GSPE. (B) ID was decreased in the SHR-C group compared with the WKY group, and this was significantly reversed by treatment with GSPE. (C) The ratio of WT/ID was significantly greater in the SHR-C group compared with the WKY and SHR-T groups. (D) WCSA levels were significantly increased in the SHR-C group compared with the control group, whereas (E) LCSA levels were significantly decreased in the SHR-C group compared with the control group. (F) The ratio of WCSA/LCSA was increased in the SHR-C group; however, this increase was significantly abrogated by treatment with GSPE. ${ }^{*} \mathrm{P}<0.05$ and ${ }^{* *} \mathrm{P}<0.01$. GSPE, grape seed proanthocyanidin extract; SHR, spontaneous hypertension rat; $\mathrm{T}$, treatment; $\mathrm{C}$, control; WKY, Wistar-Kyoto; ID, inner diameter; VCSA, vascular cross-sectional area; WCSA, wall cross-sectional area; LCSA, luminal cross-sectional area; WT, wall thickness.

calculate the ID, WT, WT/ID, WCSA, LCSA and WCSA/LCSA of small mesenteric arteries. Hypertension increased WT $(\mathrm{P}<0.01$; Fig. 4A) and decreased ID ( $\mathrm{P}<0.05$; Fig. 4B), and these effects were significantly abrogated by treatment with GSPE $(\mathrm{P}<0.05)$. Notably, the ratio of WT/ID was significantly greater in SHR-C compared with WKY and SHR-T groups $(\mathrm{P}<0.01$; Fig. 4C). Similarly, significantly increased levels of WCSA $(\mathrm{P}<0.01$; Fig. 4D) and decreased levels of LCSA $(\mathrm{P}<0.05$; Fig. 4E) were observed in the SHR-C group compared with the WKY group, and these effects were attenuated by treatment with GSPE $(\mathrm{P}<0.05)$. The ratio of WCSA/LCSA was increased in the SHR-C group, compared with the WKY control and SHR-T groups $(\mathrm{P}<0.05$; Fig. 4F).

Quantification of collagen/elastin expression and ratio. Image Pro-Plus software was used to investigate collagen and elastin expression in 5 different fields of view. Collagen expression levels were increased in the SHR-C group compared with the WKY control $(\mathrm{P}<0.01)$; this increase was abrogated by GSPE treatment $(\mathrm{P}<0.05$; Fig. 5A). Although there was no significant difference in the expression of elastin among the different groups ( $\mathrm{P}>0.05$; Fig. 5B), a significantly greater ratio of collagen/elastin was observed in the SHR-C group compared with the WKY control, which was partially reversed by treatment with GSPE ( $\mathrm{P}<0.01$; Fig. 5C).

Effect of GSPE on the expression of TGF- $\beta 1$ and collagen. The protein expression levels of collagen and TGF- $\beta 1$ were detected by western blotting, as presented in Fig. 6. The expression of collagen I and III was increased by hypertension and this increase was attenuated by GSPE treatment. In addition, TGF- $\beta 1$ expression was increased in the SHR-C group compared with the WKY group, which was abrogated by GSPE treatment.

TGF- $\beta 1$ and collagen I/III protein expression levels were normalized to $\beta$-actin levels using densitometry, as presented in Fig. 7. The expression of TGF- $\beta 1$ was significantly increased in the SHR-C group compared with the WKY group $(\mathrm{P}<0.05)$; this increase was attenuated by treatment with GSPE $(\mathrm{P}<0.01$; Fig. 7A). Significantly increased levels of collagen I $(\mathrm{P}<0.05$; Fig. 7B) and III $(\mathrm{P}<0.05$; Fig. 7C) were observed in the SHR-C group compared with the WKY group; GSPE treatment significantly reduced this hypertension-induced collagen hyperplasia $(\mathrm{P}<0.05)$. 
A

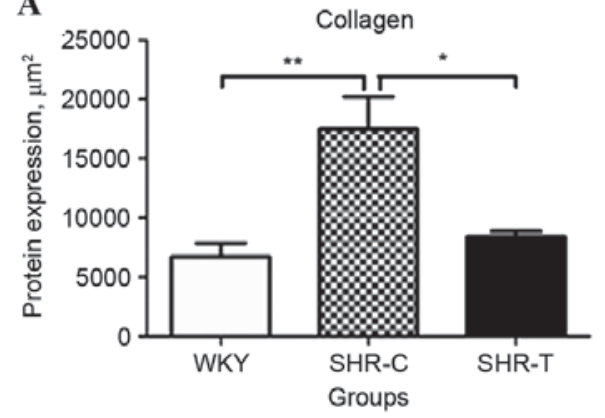

B

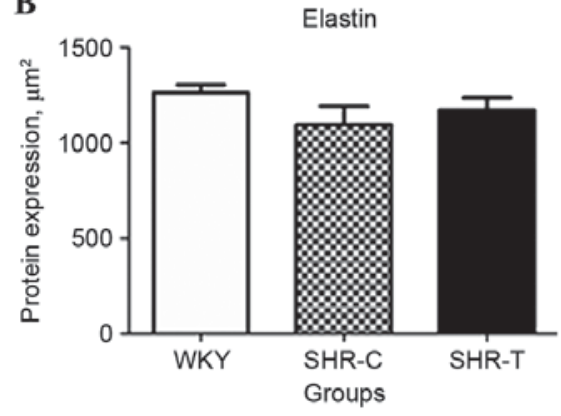

C

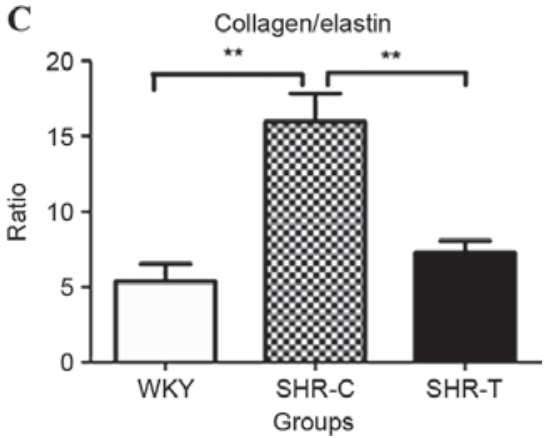

Figure 5. Effect of GSPE on the expression and ratio of collagen and elastin, as measured in Sirius red-victoria blue stained sections. (A) Expression of collagen levels increased significantly in the SHR-C group compared with the control WKY group, and this was partially reversed by treatment with GSPE. (B) Expression of elastin was not altered between groups. (C) The ratio of collagen/elastin significantly increased in the SHR-C group compared with the control WKY group; this was partially reversed by treatment with GSPE. ${ }^{*} \mathrm{P}<0.05$ and ${ }^{* *} \mathrm{P}<0.01$. GSPE, grape seed proanthocyanidin extract; SHR, spontaneous hypertension rat; T, treatment; C, control; WKY, Wistar-Kyoto.

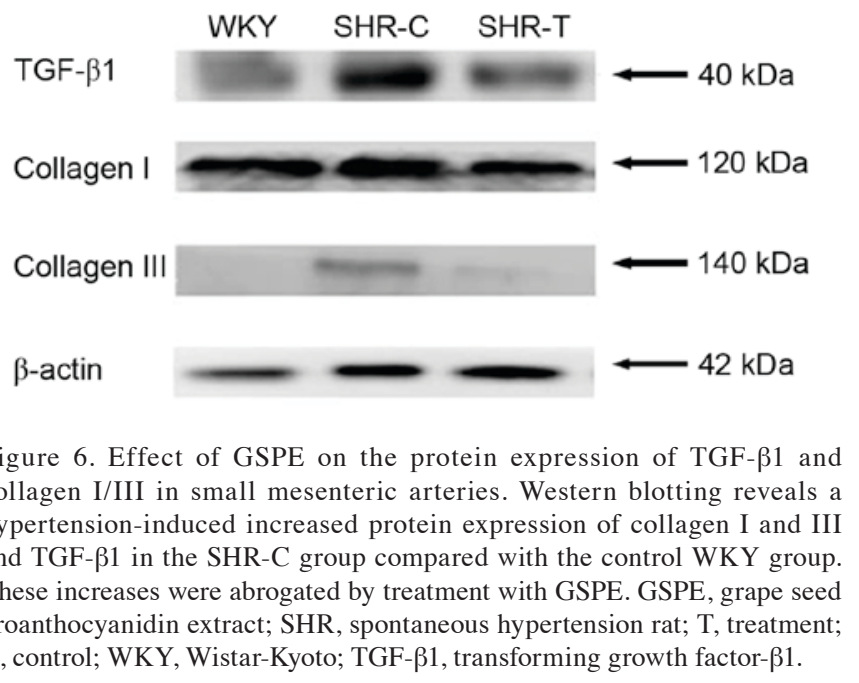

The mRNA expression levels of TGF- $\beta 1$ and collagen I/III were detected using RT-qPCR (Fig. 8). mRNA expression levels of TGF- $\beta 1$ were significantly increased by hypertension $(\mathrm{P}<0.01)$; this increase was abrogated by GSPE treatment $(\mathrm{P}<0.05$; Fig. 8A). Collagen I (Fig. 8B) and III (Fig. 8C) mRNA expression levels were upregulated in the SHR-C group compared with the WKY group $(\mathrm{P}<0.05)$; this increase was partially reversed by treatment with GSPE $(\mathrm{P}<0.05)$.

Role of GSPE in the alteration of the ultrastructure of fibroblast cells in the mesenteric small arteries. The ultrastructure of the mesenteric small artery was observed using a transmission electron microscope. In photomicrographs taken from WKY, the intima was smooth with a clear edge and collagen was homogenously distributed in the media and in adventitia (Fig. 9A). Fibroblast cells exhibited a normal morphology in the adventitia (Fig. 9B). In the SHR-C group, the inner membrane appeared to be damaged, a large quantity of irregular collagen was located in the media and invading adventitia fibroblasts (AF) appeared (Fig. 9C). In AF, the endoplasmic reticulum became dilated and the formation of a dense patch under the membrane, in addition to the development of collagen hyperplasia, were observed in the adventitia (Fig. 9D). Hypertension-induced endangium damage to the inner membrane and accumulation of collagen in the vascular wall were attenuated by GSPE (Fig. 9E). In addition, the translocation of AF into the media of the small artery, as a result of hypertension, was abrogated by GSPE. Furthermore, no dense patch was observed under the membrane, however, the endoplasmic reticulum was dilated in AFs (Fig. 9F).

\section{Discussion}

Hypertension is an important risk factor and contributes to cardiovascular diseases. Vascular remodeling, particularly in small arteries, is considered critical in the development of hypertension (13). The increased thickness of the vascular wall and the increased narrowing of the lumen are primary pathological developments in hypertension, along with the proliferation of VSMC and the accumulation of collagen in the ECM (14).

In the present study, hypertrophic vascular remodeling was observed in the small mesenteric arteries of rats with spontaneous hypertension, which presented as a reduction in the size of the lumen. This pathological reconstruction of the artery in hypertensive rats includes an increase in wall thickness, collagen overexpression and AF relocates to the media of the artery. A model of hypertension similarly demonstrated that the ID was reduced and the wall/lumen ratio was increased in small arteries as a consequence of increased wall thickness (15). The present study further confirmed that the accumulation of collagen was a primary feature that resulted in a narrowed lumen in SHR-C animals, indicated as an increase in ratio of WT/ID and WCSA/LCSA. A previous study similarly reported findings indicating that concentric remodeling occurred in humans from autopsy readings of cardiac and renal arterioles in 25 essential hypertension patients (16). An increased wall-to-lumen ratio and WCSA of retinal arterioles was observed in cerebrovascular patients compared with subjects with mild arterial hypertension (17). Thus, the critical role of vascular remodeling in the development of hypertension and the association between the increase in vascular wall thickness and the development and progression of hypertension was investigated. CSA and intima-media thickness have been considered as independently diagnostic morphological parameters, which were significantly increased 
A

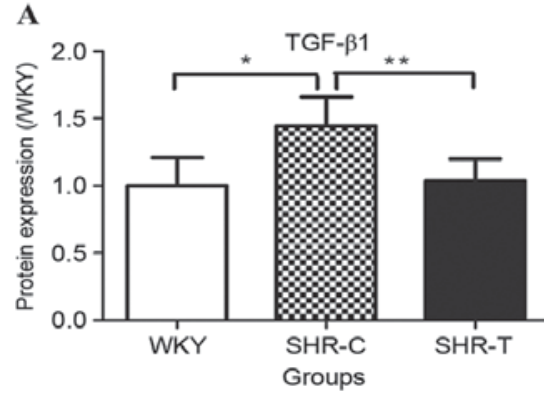

B

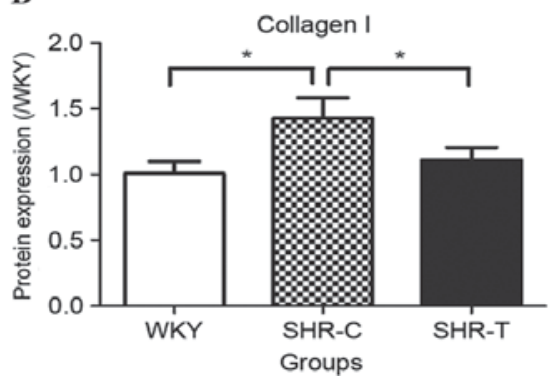

C

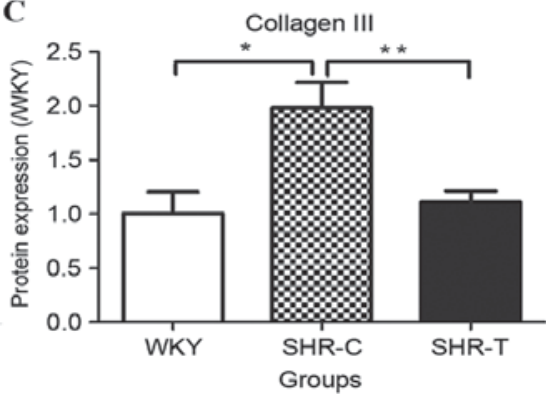

Figure 7. Quantification of the effect of GSPE on the protein expression of TGF- $\beta 1$ and collagen I/III in small mesenteric arteries. Quantification of western blotting revealed that expression of (A) TGF- $\beta 1$, (B) collagen I and (C) collagen III were significantly greater in the SHR-C group compared with the WKY control group; however, this increase was abrogated by treatment with GSPE. "P $<0.05$ and ${ }^{* *} \mathrm{P}<0.01$. GSPE, grape seed proanthocyanidin extract; SHR, spontaneous hypertension rat; T, treatment; C, control; WKY, Wistar-Kyoto; TGF- $\beta 1$, transforming growth factor- $\beta 1$.

A

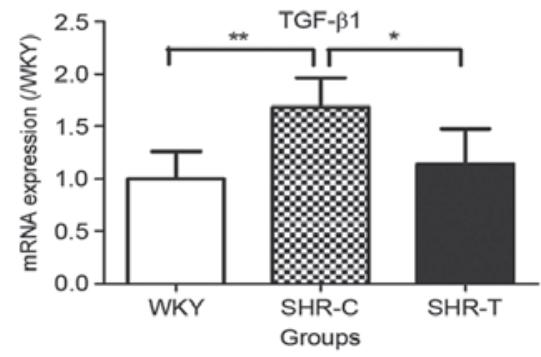

B

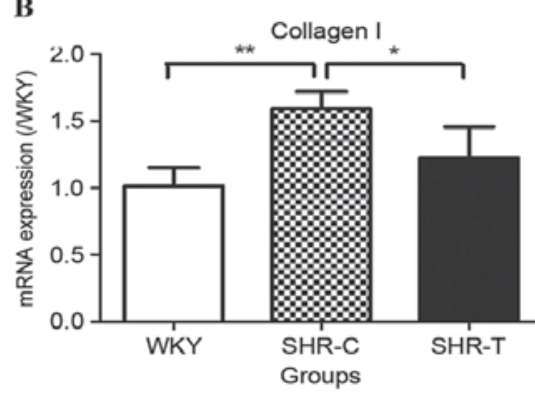

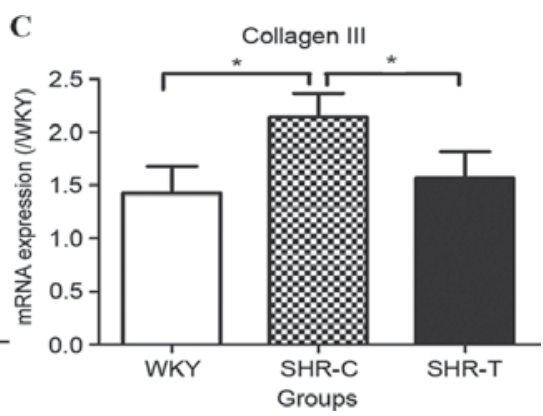

Figure 8. Effect of GSPE on mRNA expression levels of TGF- $\beta 1$ and collagen I/III in small mesenteric arteries. Reverse transcription-quantitative polymerase chain reaction revealed that (A) TGF- $\beta 1$, (B) collagen I and (C) collagen III mRNA expression levels were increased in the SHR-C group compared with the WKY control group; however, this increase was partially reversed by treatment with GSPE. ${ }^{\mathrm{P}}<0.05$ and ${ }^{* *} \mathrm{P}<0.01$. GSPE, grape seed proanthocyanidin extract; SHR, spontaneous hypertension rat; T, treatment; C, control; WKY, Wistar-Kyoto; TGF- $\beta 1$, transforming growth factor- $\beta 1$.

in patients with coronary artery disease compared with those without (18). The results of the present study suggested that hypertension resulting from hypertrophic vascular remodeling was significantly decreased by orally-administered GSPE. GSPE treatment significantly reduced wall thickness and enlarged the vascular lumen of SHR mesenteric small arteries. However, the SBP of hypertensive rats was not affected by GSPE administration. This indicated that the vascular remodeling was improved independent of decreasing blood pressure. The therapeutic benefits of GSPE on cardiovascular remodeling have been investigated through its inhibitory effect on the ROS/mitogen-activated protein kinase pathway (19). In our previous study, GSPE was examined as an antagonist of chemical-induced vascular remodeling (19).

Significant accumulation of type I and III collagen and the invasion of AF in the media were identified as contributors to the hypertrophic vascular remodeling in the present study. Morphological staining revealed that collagen had accumulated in the walls of small mesenteric arteries of hypertensive rats compared with healthy controls. However, this deregulated collagen deposition and the resulting angiostenosis appeared to be antagonized by oral administration of GSPE. The translocation of AF into the media and the proliferation of VSMC (data not shown) were observed in arterial tissues with hypertension. In addition, invading fibroblasts exhibited an altered morphology, to myofibroblasts, due to the appearance of dense paths and enlarged endoplasmic reticulum. These alterations indicated that the movement of AF, along with an associated increase in collagen, was an important factor in vascular remodeling in the development of hypertension. Previous studies have investigated the function of AF in the development of hypertension and the regulation of biopathological vascular remodeling (20). Collagen was observed to be synthesized by AF and was a predominant compound of the structural skeleton of tissues and organs (21). Notably, collagen and fibronectin were increased but elastin was decreased in the vessel wall during hypertension (13).

The results of the present study revealed that in SHR, AF metastasized into vascular media and subsequently presented a morphological transition associated with the overexpression of collagen, whereas the expression of elastin was constant. The underlying mechanism may be that oxidative stress and the subsequent generation of cytokines, such as TGF- $\beta 1$, induced AF thereby altering macrophage phenotype and invasion into the vascular wall, resulting in vascular remodeling $(22,23)$. Notably, the dysfunction resulting in the translocation of AF was reduced by GSPE treatment, which was exhibited as the endoplasmic reticulum decreasing in size and a reduction in the dense path. Oxidative stress activates a cascade of intercellular and intracellular signals, which result in functional and morphological cell alterations, which are considered to be critical in the initiation and development of hypertension $(24,25)$. Therefore, the therapeutic benefit of GSPE in vascular remodeling partly depends on its anti-oxidative ability to inhibit the pathological alteration of AF.

Oxidative stress has been widely accepted as a primary factor during the development of hypertension (26). A large body of evidence suggests that when an imbalance occurs 
A

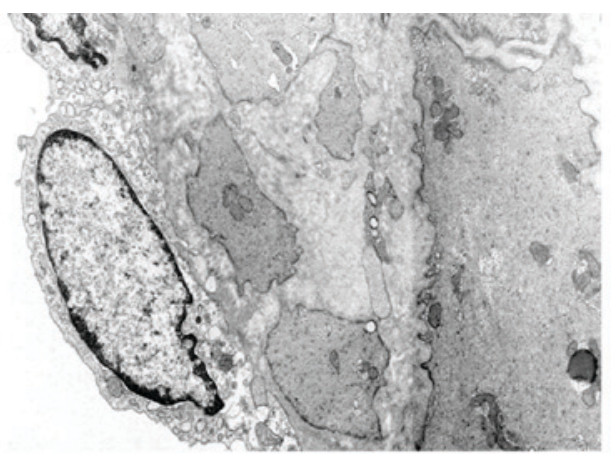

C

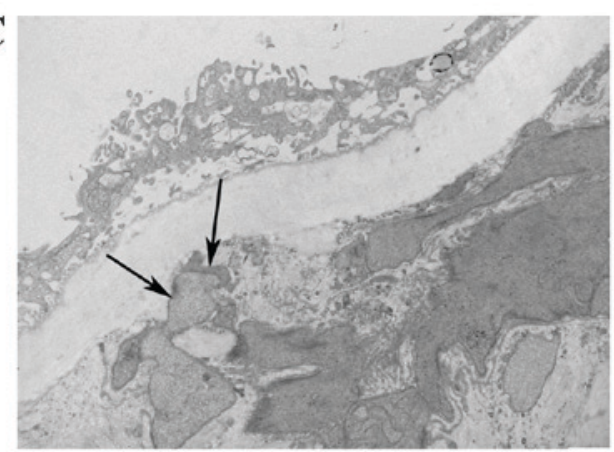

E

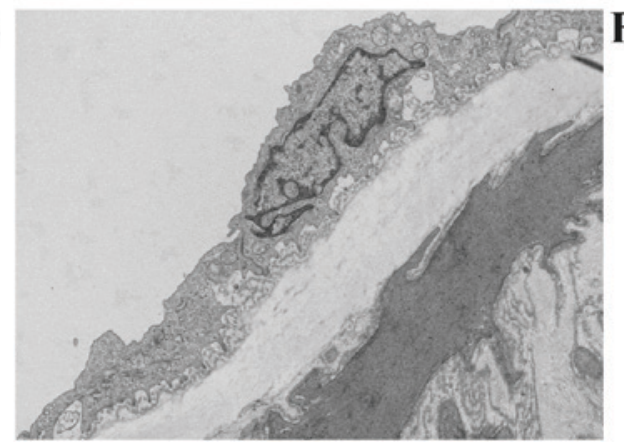

B

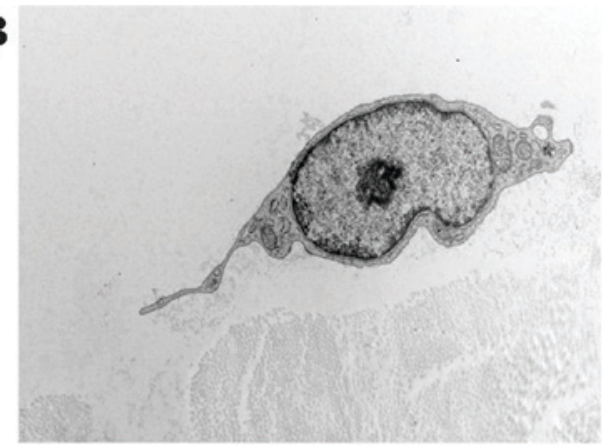

D
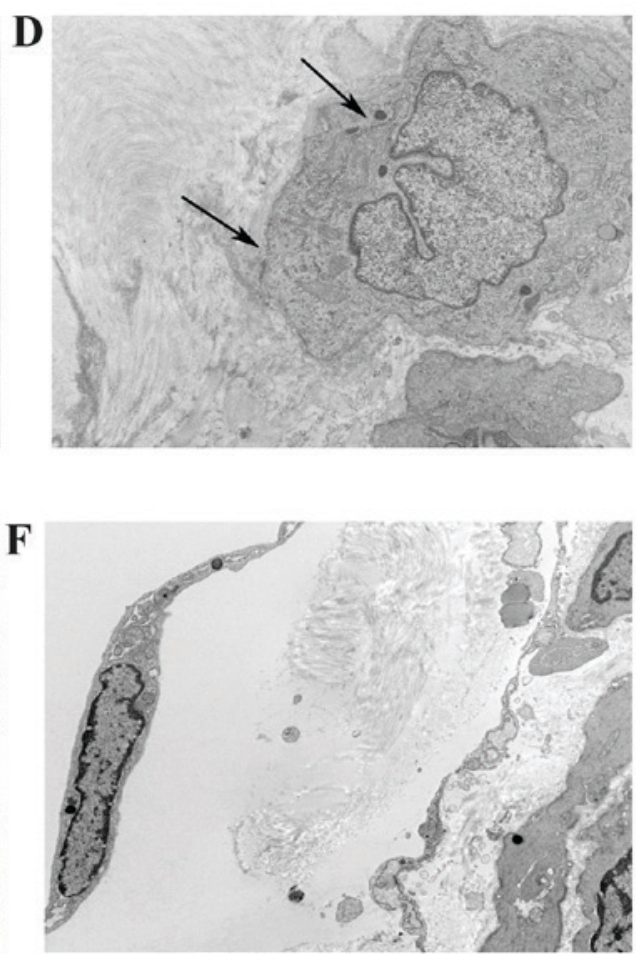

Figure 9. Effect of GSPE on ultrastructure alteration of the mesenteric small arteries. Transmission electron microscopy was used to observe the ultrastructure of the small mesenteric arteries, from (A and B) Wistar-Kyoto rats, (C and D) spontaneous hypertension control rats and (E and F) spontaneous hypertension with GSPE treatment rats. (C) The upper arrow indicates a fibroblast that has invaded the media, the lower one indicates a dense patch under the fibroblast membrane. (D) The upper arrow indicates a fibroblast in the adventitia, and the lower one indicates a dense patch under the fibroblast membrane. GSPE, grape seed proanthocyanidin extract.

between oxidant species and the antioxidant defense system, ROS is generated in the vascular system and serves as a key trigger of hypertension (27). In the present study, greater levels of ROS, MDA and $\mathrm{H}_{2} \mathrm{O}_{2}$ were detected in vascular tissue lysates, whereas reduced levels of the oxidant enzymes SOD and CAT were observed. This imbalance triggered AF translocation into the media of the mesenteric artery and subsequently resulted in an overexpression of collagen. A previous study demonstrated that a high level of ROS was observed in a sterone-induced hypertensive rat model, which was closely linked to the increased collagen deposition in the thoracic aorta (28). Behbahani et al (29) demonstrated that resveratrol from grapes rescued the dysfunction of the oxidation-reduction system in rat mesenteric small arterial tissue, via its antioxidant properties. In a controlled study involving 119 healthy, pre- and mildly hypertensive subjects, GSPE significantly decreased blood pressure and reduced the oxidation of membrane lipids (30). The data obtained from the present study suggested that the anti-oxidative effects of GSPE may result in the inhibition of morphological and functional alterations of AF; the primary contributor to vascular remodeling, and subsequently hypertension and cardiac disease.

Oxidative production activates the redox-sensitive regulation of multiple signaling pathways and second messengers, such as TGF- $\beta 1$, which responds to fibrogenic events and cell differentiation (31,32). Vascular remodeling follows a set pathway of events; ROS induces expression of angiotensin II, followed by the release of TGF- $\beta 1$, which modifies ECM composition and structure (33). TGF- $\beta 1$ subsequently enhances collagen expression in $\mathrm{AF}(34,35)$. In addition, TGF- $\beta 1$ induces the proliferation and phenotype transformation of AF, as well as their migration, and increases the expression of ECM compounds (36). The present study investigated the potential association between oxidation and TGF- $\beta 1$ elevation, the consequent ECM upregulation, and vascular wall remodeling in the small mesenteric arteries of SHR. In hypertension groups, expression levels of 
TGF- $\beta 1$ mRNA and protein were increased; however, this increase was abrogated by treatment with GSPE.

TGF- $\beta 1$ promoted the differentiation of cardiac fibroblasts into myofibroblasts and furthermore triggered collagen gel contraction by cardiac fibroblasts (37). Similar findings from the present study suggested that AF translocated into the media and this was associated with the overexpression of collagen, which may explain vascular remodeling resulting from spontaneous hypertension in rats. The present study linked the reversible effect of GSPE on vascular remodeling with the downregulation of the TGF- $\beta 1$ signaling pathway, and the subsequent prevention of AF metastasis, proliferation, phenotype alteration and the reduction of overexpression and deposition of collagen in vessels. TGF- $\beta 1$ was observed to be important in the pathogenesis of pulmonary arterial hypertension and its blockade resulted in a decrease in pulmonary vascular remodeling (38). The expression of collagen in the media of carotid arteries in SHR was stimulated by TGF- $\beta 1$ via the Smad pathway, which was blocked by the deregulation of TGF- $\beta 1$ expression (39).

In conclusion, the present study confirmed that hypertension resulted in hypertrophic vascular remodeling of the small mesenteric arteries, which was partially reversed by treatment with GSPE. The benefit of GSPE to vascular remodeling was not due to a reduction in the SBP. The novel evidence presented in the current study suggested that the anti-remodeling effect of GSPE was achieved through inhibition of ROS generation, overexpression of TGF- $\beta 1$, AF differentiation and translocation, and collagen hyperplasia in small mesenteric arteries. The anti-oxidative properties of GSPE suggested it may be a potential therapeutic agent; however, further studies are required to investigate this potential.

\section{Acknowledgements}

The present study was supported by grants from the Science and Technology Development Plan of Shandong Province (grant no. ZR2014HM071), the National Natural Science Foundation of China (grant no. 30700884), the Shandong Science and Technology Research Plan (grant no. 2010GGC10294), the Shandong Science and Technology Project Plan (grant no. 2012GB021817) and the National Science and Technology Major Project: Technology Platform Construction for Clinical Evaluation of Cardiovascular New Drug (grant no. 2012ZX09303016-003).

\section{References}

1. Wang M, Kim SH, Monticone RE and Lakatta EG: Matrix metalloproteinases promote arterial remodeling in aging, hypertension, and atherosclerosis. Hypertension 6: 698-703, 2015.

2. van Guldener C and Stehouwer CD: Hyperhomocysteinemia, vascular pathology, and endothelial dysfunction. Semin Thromb Hemost 26: 281-289, 2000.

3. Ponticos M and Smith BD: Extracellular matrix synthesis in vascular disease: Hypertension and atherosclerosis. J Biomed Res 28: 25-39, 2014.

4. Rodrigo R, Passalacqua W, Araya J, Orellana M and Rivera G: Implications of oxidative stress and homocysteine in the pathophysiology of essential hypertension. J Cardiovasc Pharmacol 42: 453-461, 2003.

5. Lijnen P, Papparella I, Petrov V, Semplicini A and Fagard R: Angiotensin II-stimulated collagen production in cardiac fibroblasts is mediated by reactive oxygen species. J Hypertens 24 757-766, 2006.
6. Shi Y,Pieniek M, Fard A, O'Brien J, Mannion JD and Zalewski A: Adventitial remodeling after coronary arterial injury. Circulation 93: 340-348, 1996.

7. Shen WL, Gao PJ, Che ZQ, Ji KD, Yin M, Yan C, Berk BC and Zhu DL: NAD $(\mathrm{P}) \mathrm{H}$ oxidase-derived reactive oxygen species regulate angiotensin-II induced adventitial fibroblast phenotypic differentiation.BiochemBiophysRes Commun339:337-343,2006.

8. Holmström KM and Finkel T: Cellular mechanisms and physiological consequences of redox-dependent signalling. Nat Rev Mol Cell Biol 15: 411-421, 2014.

9. Sharifi AM, Li JS, Endemann D and Schiffrin EL: Effects of enalapril and amlodipine on small-artery structure and composition, and on endothelial dysfunction in spontaneously hypertensive rats. J Hypertens 16: 457-466, 1998.

10. Junqueira LC, Cossermelli W and Brentani R: Differential staining of collagens type I, II and III by Sirius Red and polarization microscopy. Arch Histol Jpn 41: 267-274, 1978.

11. Livak KJ and Schmittgen TD: Analysis of relative gene expression data using real-time quantitative PCR and the 2(-Delta Delta C(T)) method. Methods 25: 402-408, 2001.

12. Jun L, Yuanshu W, Yanying X, Zhongfa X, Jian Y, Fengling W, Xianjun Q, Kokudo N, Wei T, Weixia Z and Shuxiang C: Altered mRNA expressions of sialyltransferases in human gastric cancers. Med Oncol 29: 84-90, 2012

13. Rattazzi M, Bertacco E, Puato M, Faggin E and Pauletto P: Hypertension and vascular calcification: A vicious cycle? J Hypertens 30: 1885-1893, 2012.

14. Gündüz F, Baskurt OK and Meiselman HJ: Vascular dilation responses of rat small mesenteric arteries at high intravascular pressure in spontaneously hypertensive rats. Circ J 73: 2091-2097, 2009.

15. Zhang E, Maruyama J, Yokochi A, Mitani Y, Sawada H, Nishikawa M, Ma N and Maruyama K: Sarpogrelate hydrochloride, a serotonin $5 \mathrm{HT} 2 \mathrm{~A}$ receptor antagonist, ameliorates the development of chronic hypoxic pulmonary hypertension in rats. J Anesth 29: 715-723, 2015.

16. Pei F, Li XY, Fang Y, Shi HY and Diao HJ: Cardiac and renal arteriolar pathological changes in the autopsied elderly hypertensive patients with left ventricular hypertrophy. Zhonghua Xin Xue Guan Bing Za Zhi 36: 872-877, 2008 (In Chinese).

17. Baleanu D, Ritt M, Harazny J, Heckmann J, Schmieder RE and Michelson G: Wall-to-lumen ratio of retinal arterioles and arteriole-to-venule ratio of retinal vessels in patients with cerebrovascular damage. Invest Ophthalmol Vis Sci 50: 4351-4359, 2009.

18. Frick M, Schwarzacher SP, Alber HF, Rinner A, Ulmer H, Pachinger $\mathrm{O}$ and Weidinger F: Morphologic rather than functional or mechanical sonographic parameters of the brachial artery are related to angiographically evident coronary atherosclerosis. J Am Coll Cardiol 40: 1825-1830, 2002.

19. Huang LL, Pan C, Wang L, Ding L, Guo K, Wang HZ, Xu AM and Gao S: Protective effects of grape seed proanthocyanidins on cardiovascular remodeling in DOCA-salt hypertension rats. J Nutr Biochem 26: 841-849, 2015.

20. Liu X, Qiu J, Zhao S, You B, Ji X, Wang Y, Cui X, Wang Q and Gao H: Grape seed proanthocyanidin extract alleviates ouabain-induced vascular remodeling through regulation of endothelial function. Mol Med Rep 6: 949-954, 2012.

21. Arteaga-Solis E, Gayraud B and Ramirez F: Elastic and collagenous networks in vascular diseases. Cell Struct Funct 25: 69-72, 2000.

22. Siow RC, Mallawaarachchi CM and Weissberg PL: Migration of adventitial myofibroblasts following vascular balloon injury: Insights from in vivo gene transfer to rat carotid arteries. Cardiovasc Res 59: 212-221, 2003.

23. Stenmark KR, Yeager ME, El Kasmi KC, Nozik-Grayck E, Gerasimovskaya EV, Li M, Riddle SR and Frid MG: The adventitia: Essential regulator of vascular wall structure and function. Annu Rev Physiol 75: 23-47, 2013.

24. Siow RC and Churchman AT: Adventitial growth factor signalling and vascular remodelling: Potential of perivascular gene transfer from the outside-in. Cardiovasc Res 75: 659-668, 2007.

25. Barnes JL and Gorin Y: Myofibroblast differentiation during fibrosis: Role of NAD(P)H oxidases. Kidney Int 79: 944-956, 2011.

26. Montezano AC, Dulak-Lis M, Tsiropoulou S, Harvey A, Briones AM and Touyz RM: Oxidative stress and human hypertension: Vascular mechanisms, biomarkers, and novel therapies. Can J Cardiol 31: 631-641, 2015. 
27. Brito R, Castillo G, González J, Valls N and Rodrigo R: Oxidative stress in hypertension: Mechanisms and therapeutic opportunities. Exp Clin Endocrinol Diabetes 123: 325-335, 2015.

28. Chen QZ, Han WQ, Chen J, Zhu DL, Chen-Yan and Gao PJ: Anti-stiffness effect of apocynin in deoxycorticosterone acetate-salt hypertensive rats via inhibition of oxidative stress. Hypertens Res 36: 306-312, 2013.

29. Behbahani J, Thandapilly SJ, Louis XL, Huang Y, Shao Z, Kopilas MA, Wojciechowski P, Netticadan T and Anderson HD: Resveratrol and small artery compliance and remodeling in the spontaneously hypertensive rat. Am J Hypertens 23: 1273-1278, 2010.

30. Belcaro G, Ledda A, Hu S, Cesarone MR, Feragalli B and Dugall M: Grape Seed Procyanidins in Pre- and Mild Hypertension: A registry study. Evid Based Complement Alternat Med 2013: 313142, 2013.

31. Purnomo Y, Piccart Y, Coenen T, Prihadi JS and Lijnen PJ Oxidative stress and transforming growth factor- $\beta 1$-induced cardiac fibrosis. Cardiovasc Hematol Disord Drug Targets 13: $165-172,2013$

32. Lin Z, Liu L, Xi Z, Huang J and Lin B: Single-walled carbon nanotubes promote rat vascular adventitial fibroblasts to transform into myofibroblasts by SM22- $\alpha$ expression. Int J Nanomedicine 7: 4199-4206, 2012.

33. Yoshioka J, Schreiter ER and Lee RT: Role of thioredoxin in cell growth through interactions with signaling molecules. Antioxid Redox Signal 8: 2143-2151, 2006.
34. Ding WJ and Hong L: Progress research on relationship between oxidative stress and collagen metabolism diseases. Journal of Jilin University (Medicine Edition) 39: 1302-1306, 2013.

35. Silacci $\mathrm{P}$ and Hayoz D: Oxidative stress as the triggering event for vascular remodelling. Nephrol Dial Transplant 13: 1343-1346, 1998.

36. Takimoto E and Kass DA: Role of oxidative stress in cardiac hypertrophy and remodeling. Hypertension 49: 241-248, 2007.

37. Lijnen P, Petrov V, Rumilla K and Fagard R: Transforming growth factor-beta 1 promotes contraction of collagen gel by cardiac fibroblasts through their differentiation into myofibroblasts. Methods Find Exp Clin Pharmacol 25: 79-86, 2003.

38. Graham BB, Chabon J, Gebreab L, Poole J, Debella E, Davis L, Tanaka T, Sanders L, Dropcho N, Bandeira A, et al: Transforming growth factor- $\beta$ signaling promotes pulmonary hypertension caused by Schistosoma mansoni. Circulation 128: 1354-1364, 2013.

39. Chen JL, Shang QH, Hu W, Liu C, Mao WH and Liu HQ: Role of TGF- $\beta 1 /$ Smads pathway in carotid artery remodeling in renovascular hypertensive rats and prevention by Enalapril and Amlodipine. J Geriatr Cardiol 9: 185-191, 2012. 